\title{
Direct and converse theorems for King operators
}

\author{
Zoltán Finta \\ Babeş-Bolyai University, \\ Department of Mathematics, \\ 1, M. Kogălniceanu st., \\ 400084 Cluj-Napoca, Romania \\ email: fzoltan@math.ubbcluj.ro
}

\begin{abstract}
For the sequence of King operators, we establish a direct approximation theorem via the first order Ditzian-Totik modulus of smoothness, and a converse approximation theorem of Berens-Lorentz-type.
\end{abstract}

\section{Introduction}

Studying the connection between regular summability matrices and convergent positive linear operators, King [3] introduced an interesting Bernstein-type operator defined as follows:

$$
\left(V_{n} f\right)(x) \equiv V_{n}(f ; x)=\sum_{k=0}^{n} p_{n, k}\left(r_{n}(x)\right) f\left(\frac{k}{n}\right),
$$

where $x \in[0,1], f \in C[0,1], p_{n, k}(x)=\left(\begin{array}{l}n \\ k\end{array}\right) x^{k}(1-x)^{n-k}$ and

$$
r_{n}(x)=\left\{\begin{array}{rll}
x^{2}, & \text { if } & n=1 \\
-\frac{1}{2(n-1)}+\sqrt{\frac{n}{n-1} x^{2}+\frac{1}{4(n-1)^{2}},} & \text { if } & n=2,3, \ldots
\end{array}\right.
$$

2010 Mathematics Subject Classification: 41A10, 41A25, 41A36

Key words and phrases: Bernstein operators, King operators, first order Ditzian-Totik modulus of smoothness, Berens-Lorentz lemma 
For $r_{n}(x)=x, x \in[0,1]$, we recover from (1) the classical Bernstein operator:

$$
\left(B_{n} f\right)(x) \equiv B_{n}(f ; x)=\sum_{k=0}^{n} p_{n, k}(x) f\left(\frac{k}{n}\right) .
$$

It is known that

$$
\left(B_{n} e_{0}\right)(x)=1, \quad\left(B_{n} e_{1}\right)(x)=x \quad \text { and } \quad\left(B_{n} e_{2}\right)(x)=x^{2}+\frac{x(1-x)}{n},
$$

where $e_{j}(x)=x^{j}, x \in[0,1]$ and $j \in\{0,1,2, \ldots\}$. In contrast with (3), we have for $V_{n}$ the relations (see [3, pp. 204-205]):

$$
\left(V_{n} e_{0}\right)(x)=1, \quad\left(V_{n} e_{1}\right)(x)=r_{n}(x) \quad \text { and } \quad\left(V_{n} e_{2}\right)(x)=x^{2}
$$

The goal of the paper is to obtain direct and converse approximation theorems for the operators given by (1)-(2). The direct result is established with the aid of the first order Ditzian-Totik modulus of smoothness defined by

$$
\omega_{\varphi}^{1}(f ; \delta)=\sup _{0<h \leq \delta} \sup _{x \pm \frac{1}{2} h \varphi(x) \in[0,1]}\left|f\left(x+\frac{1}{2} h \varphi(x)\right)-f\left(x-\frac{1}{2} h \varphi(x)\right)\right|,
$$

where $\varphi(x)=\sqrt{x(1-x)}, x \in[0,1]$. It is known [2, Theorem 2.1.1] that (5) is equivalent with the K-functional

$$
K_{1, \varphi}(f ; \delta)=\inf _{g \in W(\varphi)}\left\{\|f-g\|+\delta\left\|\varphi g^{\prime}\right\|\right\}, \quad \delta>0,
$$

where $W(\varphi)=\left\{g \mid g \in A . C \cdot{ }_{\text {loc }}[0,1],\left\|\varphi g^{\prime}\right\|<\infty\right\}$, i.e. there exists $C_{1}>0$ such that

$$
C_{1}^{-1} \omega_{\varphi}^{1}(f ; \delta) \leq K_{1, \varphi}(f ; \delta) \leq C_{1} \omega_{\varphi}^{1}(f ; \delta) .
$$

Finally, a converse result of Berens-Lorentz-type is established for the operators $V_{n}$ (see [1, p. 312, Lemma 5.2] and Lemma 3 below). Throughout this paper $\mathrm{C}_{1}, \mathrm{C}_{2}, \ldots, \mathrm{C}_{13}$ denote absolute positive constants.

\section{Direct theorem}

We have the following result for the functions defined by (2).

Lemma 1 The functions $r_{n}, n=1,2, \ldots$, satisfy the properties 
a) $0 \leq r_{n}^{\prime}(x) \leq 2$ for $x \in[0,1]$ and $n=1,2, \ldots$;

b) $r_{n}(0)=0, r_{n}(1)=1$ and $r_{n}$ is strictly increasing function on $[0,1]$ for $n=1,2, \ldots$;

c) $0 \leq r_{n}(x) \leq x \leq 1$ for $x \in[0,1]$ and $n=1,2, \ldots$;

d) $0 \leq x-r_{n}(x) \leq \frac{2}{n}(1-x)$ for $x \in[0,1]$ and $n=1,2, \ldots$;

e) $x \leq 2 r_{n}(x)$ for $x \in\left[\frac{1}{n}, 1\right]$ and $n=1,2, \ldots$

Proof. a) Obviously $r_{1}^{\prime}(x)=2 x, x \in[0,1]$. For $n \geq 2$, by simple computations, we obtain

$$
r_{n}^{\prime}(x)=\left\{\begin{array}{cll}
\lim _{x \searrow 0} \frac{r_{n}(x)-r_{n}(0)}{x-0}=0, & \text { if } x=0 \\
\frac{\frac{n}{n-1} x}{\sqrt{\frac{n}{n-1} x^{2}+\frac{1}{4(n-1)^{2}}}}, & \text { if } & 0<x \leq 1 .
\end{array}\right.
$$

Hence $0 \leq r_{n}^{\prime}(x) \leq \frac{\frac{n}{n-1} x}{\sqrt{\frac{n}{n-1}} x}=\sqrt{\frac{n}{n-1}} \leq \sqrt{2}$ for $x \in(0,1]$. Thus $0 \leq r_{n}^{\prime}(x) \leq 2$ for $n=1,2, \ldots$ and $x \in[0,1]$.

b) It follows from (2) and a).

c) It follows from (2) by direct computations.

d) Obviously $0 \leq x-r_{1}(x)=x(1-x) \leq 2(1-x), x \in[0,1]$. Using $\left.b\right)$ and c), we have $0 \leq x-r_{n}(x) \leq \frac{2}{n}(1-x)$ for $x=0$ and $n \geq 2$, and

$$
\begin{aligned}
0 \leq x-r_{n}(x) & =x+\frac{1}{2(n-1)}-\sqrt{\frac{n}{n-1} x^{2}+\frac{1}{4(n-1)^{2}}} \\
& =\frac{\frac{x(1-x)}{n-1}}{x+\frac{1}{2(n-1)}+\sqrt{\frac{n}{n-1} x^{2}+\frac{1}{4(n-1)^{2}}}} \\
& \leq \frac{\frac{x(1-x)}{n-1}}{x}=\frac{1-x}{n-1} \leq \frac{2}{n}(1-x)
\end{aligned}
$$

for $x \in(0,1]$ and $n \geq 2$.

e) For $n=1$ the statement is obvious. For $n \geq 2$, we consider the function $h(x)=\frac{x}{r_{n}(x)}, x \in\left[\frac{1}{n}, 1\right]$. Then, by $(2)$,

$$
h^{\prime}(x)=\frac{r_{n}(x)-x r_{n}^{\prime}(x)}{r_{n}^{2}(x)}=r_{n}^{-2}(x)\left(r_{n}(x)+\frac{1}{2(n-1)}\right)^{-1 / 2}
$$




$$
\times \frac{1}{2(n-1)}\left[\frac{1}{2(n-1)}-\sqrt{\frac{n}{n-1} x^{2}+\frac{1}{4(n-1)^{2}}}\right]<0
$$

for $x \in\left[\frac{1}{n}, 1\right]$. Hence

$$
\begin{aligned}
h(x) & \leq h\left(\frac{1}{n}\right)=\frac{\frac{1}{n}}{-\frac{1}{2(n-1)}+\sqrt{\frac{n}{n-1} \frac{1}{n^{2}}+\frac{1}{4(n-1)^{2}}}} \\
& =\frac{2(n-1)}{\sqrt{n}} \frac{1}{\sqrt{5 n-4}-\sqrt{n}}=\frac{1}{2} \frac{\sqrt{5 n-4}+\sqrt{n}}{\sqrt{n}} \leq 2
\end{aligned}
$$

for $x \in\left[\frac{1}{n}, 1\right]$, which was to be proved.

The operators $V_{n}$ given by (1)-(2) are linear and positive. By Lemma $\left.1, b\right)$, we have

$$
\left(V_{n} f\right)(0)=f(0) \quad \text { and } \quad\left(V_{n} f\right)(1)=f(1)
$$

for all $f \in C[0,1]$.

In the next theorem we establish the direct result.

Theorem 1 There exists $\mathrm{C}_{2}>0$ such that

$$
\left\|V_{n} f-f\right\| \leq C_{2} \omega_{\varphi}^{1}\left(f ; \frac{1}{\sqrt{n}}\right)
$$

for all $\mathrm{f} \in \mathrm{C}[0,1]$ and $\mathrm{n}=1,2, \ldots$

Proof. Let $x \in(0,1)$ and $t \in[0,1]$. Taking into account [2, Lemma 9.6.1], we have

$$
\left|\int_{x}^{t} \frac{d u}{\varphi(u)}\right| \leq \varphi^{-1}(x)|t-x|^{1 / 2}\left|\int_{x}^{t} \frac{d u}{|t-u|^{1 / 2}}\right|=2 \varphi^{-1}(x)|t-x| .
$$

Further, for $g \in W(\varphi)$, we have $g(t)=g(x)+\int_{x}^{t} g^{\prime}(u) d u, t \in[0,1]$ and $x \in(0,1)$. Hence, by (9), Hölder's inequality, (4) and Lemma 1 , d), we get

$$
\begin{aligned}
& \left|V_{n}(g ; x)-g(x)\right|=\left|V_{n}\left(\int_{x}^{t} g^{\prime}(u) d u ; x\right)\right| \\
& \quad \leq V_{n}\left(\left|\int_{x}^{t}\right| g^{\prime}(u)|d u| ; x\right) \leq\left\|\varphi g^{\prime}\right\| V_{n}\left(\left|\int_{x}^{t} \frac{d u}{\varphi(u)} d u\right| ; x\right)
\end{aligned}
$$




$$
\begin{aligned}
& \leq 2 \varphi^{-1}(x)\left\|\varphi g^{\prime}\right\| V_{n}(|t-x| ; x) \leq 2 \varphi^{-1}(x)\left\|\varphi g^{\prime}\right\|\left(V_{n}\left((t-x)^{2} ; x\right)\right)^{1 / 2} \\
& =2 \varphi^{-1}(x)\left\|\varphi g^{\prime}\right\|\left(V_{n}\left(e_{2} ; x\right)-2 x V_{n}\left(e_{1} ; x\right)+x^{2} V_{n}\left(e_{0} ; x\right)\right)^{1 / 2} \\
& =2 \varphi^{-1}(x)\left\|\varphi g^{\prime}\right\|\left(x^{2}-2 x r_{n}(x)+x^{2}\right)^{1 / 2}=2 \varphi^{-1}(x)\left\|\varphi g^{\prime}\right\|\left(2 x\left(x-r_{n}(x)\right)\right)^{1 / 2} \\
& \leq 2 \varphi^{-1}(x)\left\|\varphi g^{\prime}\right\|\left(2 x \frac{2}{n}(1-x)\right)^{1 / 2}=\frac{4}{\sqrt{n}}\left\|\varphi g^{\prime}\right\| .
\end{aligned}
$$

Due to (7), the estimation (10) is also valid for $x \in\{0,1\}$.

On the other hand, by (4), we obtain $\left|\left(V_{n} f\right)(x)\right| \leq \sum_{k=0}^{n} p_{n, k}\left(r_{n}(x)\right)\left|f\left(\frac{k}{n}\right)\right| \leq$ $\|f\| \sum_{k=0}^{n} p_{n, k}\left(r_{n}(x)\right) \leq\|f\|$, therefore

$$
\left\|V_{n} f\right\| \leq\|f\|
$$

for all $f \in C[0,1]$.

Now, in view of (4), (10) and (11), we find that

$$
\begin{aligned}
\left|V_{n}(f ; x)-f(x)\right| & \leq\left|V_{n}(f-g ; x)\right|+\left|V_{n}(g ; x)-g(x)\right|+|g(x)-f(x)| \\
& \leq 2\|f-g\|+\frac{4}{\sqrt{n}}\left\|\varphi g^{\prime}\right\| \leq 4\left\{\|f-g\|+\frac{1}{\sqrt{n}}\left\|\varphi g^{\prime}\right\|\right\} .
\end{aligned}
$$

Taking the infimum on the right hand side over all $g \in W(\varphi)$, we obtain

$$
\left\|V_{n} f-f\right\| \leq 4 K_{1, \varphi}\left(f ; \frac{1}{\sqrt{n}}\right) .
$$

Hence, by (6), we arrive at (8), which completes the proof.

\section{Converse theorem}

We begin with the following remark.

Remark 1 Due to (8), the condition $\omega_{\varphi}^{1}(f ; \delta) \leq \mathrm{C}_{3} \delta^{\alpha}, \delta>0,0<\alpha<1$ implies that $\left\|\mathrm{V}_{\mathrm{n}} \mathrm{f}-\mathrm{f}\right\| \leq \mathrm{C}_{4} \mathrm{n}^{-\alpha / 2}, \mathrm{n} \geq 1$.

In what follows, we establish the converse result of the statement given in Remark 1. To achieve this we need some lemmas. 


\section{Lemma 2 We have}

a) $\left\|\varphi\left(V_{n} f\right)^{\prime}\right\| \leq 8 \sqrt{n}\|f\|$ for $f \in C[0,1]$ and $n=1,2, \ldots$;

b) $\left\|\varphi\left(\mathrm{V}_{\mathrm{n}} \mathrm{g}\right)^{\prime}\right\| \leq 32\left\|\varphi \mathrm{g}^{\prime}\right\|$ for $\mathrm{g} \in \mathrm{W}(\varphi)$ and $\mathrm{n}=1,2, \ldots$

Proof. a) Let $x \in(0,1)$. By [1, p. 305, (2.1)], we have for the derivatives of $p_{n, k}$ that

$$
p_{n, k}^{\prime}(x)=n\left[p_{n-1, k-1}(x)-p_{n-1, k}(x)\right]=\varphi^{-2}(x)(k-n x) p_{n, k}(x),
$$

where $k=1,2, \ldots, n$ and $p_{n-1,-1}(x)=p_{n-1, n}(x)=0$. We distinguish two cases: $x \in\left(0, \frac{1}{n}\right]$. By (1), (12), Lemma 1, a) and (4), we get

$$
\begin{aligned}
\left|\varphi(x)\left(V_{n} f\right)^{\prime}(x)\right| & =\varphi(x) r_{n}^{\prime}(x)\left|\sum_{k=0}^{n} p_{n, k}^{\prime}\left(r_{n}(x)\right) f\left(\frac{k}{n}\right)\right| \\
& =n \varphi(x) r_{n}^{\prime}(x)\left|\sum_{k=0}^{n}\left[p_{n-1, k-1}\left(r_{n}(x)\right)-p_{n-1, k}\left(r_{n}(x)\right)\right] f\left(\frac{k}{n}\right)\right| \\
& =n \varphi(x) r_{n}^{\prime}(x)\left|\sum_{k=0}^{n-1} p_{n-1, k}\left(r_{n}(x)\right)\left[f\left(\frac{k+1}{n}\right)-f\left(\frac{k}{n}\right)\right]\right| \\
& \leq n \varphi(x) r_{n}^{\prime}(x) \sum_{k=0}^{n-1} p_{n-1, k}\left(r_{n}(x)\right)\left|f\left(\frac{k+1}{n}\right)-f\left(\frac{k}{n}\right)\right| \\
& \leq 2 n \varphi(x) r_{n}^{\prime}(x)\|f\| \sum_{k=0}^{n-1} p_{n-1, k}\left(r_{n}(x)\right) \leq 4 n \sqrt{x(1-x)}\|f\| \\
& \leq 4 \sqrt{n}\|f\| .
\end{aligned}
$$

$x \in\left[\frac{1}{n}, 1\right)$. Using (1), Lemma 1, a), (12), Hölder's inequality, (4) and Lemma

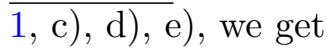

$$
\begin{aligned}
& \left|\varphi(x)\left(V_{n} f\right)^{\prime}(x)\right|=\varphi(x) r_{n}^{\prime}(x)\left|\sum_{k=0}^{n} p_{n, k}^{\prime}\left(r_{n}(x)\right) f\left(\frac{k}{n}\right)\right| \\
& \leq 2 \varphi(x)\|f\| \sum_{k=0}^{n} \varphi^{-2}\left(r_{n}(x)\right)\left|k-n r_{n}(x)\right| p_{n, k}\left(r_{n}(x)\right) \\
& \leq 2 \varphi(x) \varphi^{-2}\left(r_{n}(x)\right)\|f\|\left(\sum_{k=0}^{n}\left(k-n r_{n}(x)\right)^{2} p_{n, k}\left(r_{n}(x)\right)\right)^{1 / 2}
\end{aligned}
$$




$$
\begin{aligned}
& =2 n \varphi(x) \varphi^{-2}\left(r_{n}(x)\right)\|f\|\left(V_{n}\left(e_{2} ; x\right)-2 r_{n}(x) V_{n}\left(e_{1} ; x\right)+r_{n}^{2}(x) V_{n}\left(e_{0} ; x\right)\right)^{1 / 2} \\
& =2 n \varphi(x) \varphi^{-2}\left(r_{n}(x)\right)\|f\|\left(x^{2}-2 r_{n}^{2}(x)+r_{n}^{2}(x)\right)^{1 / 2} \\
& =2 n \varphi(x) \varphi^{-2}\left(r_{n}(x)\right)\|f\|\left(x+r_{n}(x)\right)^{1 / 2}\left(x-r_{n}(x)\right)^{1 / 2} \\
& \leq 2 n \varphi(x) \varphi^{-2}\left(r_{n}(x)\right)\|f\| \sqrt{2 x} \sqrt{\frac{2}{n}(1-x)}=4 \sqrt{n} \frac{\varphi^{2}(x)}{\varphi^{2}\left(r_{n}(x)\right)}\|f\| \\
& =4 \sqrt{n} \frac{x}{r_{n}(x)} \frac{1-x}{1-r_{n}(x)}\|f\| \leq 4 \sqrt{n} \cdot 2 \cdot 1 \cdot\|f\|=8 \sqrt{n}\|f\|
\end{aligned}
$$

Finally, by Lemma 1, a), we get $\varphi(0)\left(V_{n} f\right)^{\prime}(0)=\varphi(0) \sum_{k=0}^{n} p_{n, k}\left(r_{n}(0)\right) r_{n}^{\prime}(0) f\left(\frac{k}{n}\right)$ $=0$ and $\varphi(1)\left(V_{n} f\right)^{\prime}(1)=\varphi(1) \sum_{k=0}^{n} p_{n, k}\left(r_{n}(1)\right) r_{n}^{\prime}(1) f\left(\frac{k}{n}\right)=0$. Hence, due to (13) and (14), we obtain $\left\|\varphi\left(V_{n} f\right)^{\prime}\right\| \leq 8 \sqrt{n}\|f\|$, which was to be proved.

b) The proof is similar to the above. Let $x \in\left(0, \frac{1}{n}\right]$. Taking into account (1), (12), Lemma 1, a), (9), Hölder's inequality and (4), we get for $g \in W(\varphi)$ that

$$
\begin{aligned}
& \left|\left(V_{n} g\right)^{\prime}(x)\right|=n r_{n}^{\prime}(x)\left|\sum_{k=0}^{n}\left[p_{n-1, k-1}\left(r_{n}(x)\right)-p_{n-1, k}\left(r_{n}(x)\right)\right] g\left(\frac{k}{n}\right)\right| \\
& =n r_{n}^{\prime}(x)\left|\sum_{k=0}^{n-1} p_{n-1, k}\left(r_{n}(x)\right)\left[g\left(\frac{k+1}{n}\right)-g\left(\frac{k}{n}\right)\right]\right| \\
& \leq 2 n \sum_{k=0}^{n-1} p_{n-1, k}\left(r_{n}(x)\right)\left|g\left(\frac{k+1}{n}\right)-g\left(\frac{k}{n}\right)\right| \\
& \leq 2 n \sum_{k=0}^{n-1} p_{n-1, k}\left(r_{n}(x)\right)\left\{\left|g\left(\frac{k+1}{n}\right)-g(x)\right|+\left|g\left(\frac{k}{n}\right)-g(x)\right|\right\} \\
& \leq 2 n \sum_{k=0}^{n-1} p_{n-1, k}\left(r_{n}(x)\right)\left\{\left|\int_{x}^{\frac{k+1}{n}}\right| g^{\prime}(u)|d u|+\left|\int_{x}^{\frac{k}{n}}\right| g^{\prime}(u)|d u|\right\} \\
& \leq 2 n\left\|\varphi g^{\prime}\right\| \sum_{k=0}^{n-1} p_{n-1, k}\left(r_{n}(x)\right)\left\{\left|\int_{x}^{\frac{k+1}{n}} \frac{d u}{\varphi(u)}\right|+\left|\int_{x}^{\frac{k}{n}} \frac{d u}{\varphi(u)}\right|\right\} \\
& \leq 4 n \varphi^{-1}(x)\left\|\varphi g^{\prime}\right\| \sum_{k=0}^{n-1} p_{n-1, k}\left(r_{n}(x)\right)\left\{\left|\frac{k+1}{n}-x\right|+\left|\frac{k}{n}-x\right|\right\}
\end{aligned}
$$




$$
\begin{aligned}
\leq & 4 n \varphi^{-1}(x)\left\|\varphi g^{\prime}\right\|\left\{\left(\sum_{k=0}^{n-1} p_{n-1, k}\left(r_{n}(x)\right)\left(\frac{k+1}{n}-x\right)^{2}\right)^{1 / 2}\right. \\
& \left.+\left(\sum_{k=0}^{n-1} p_{n-1, k}\left(r_{n}(x)\right)\left(\frac{k}{n}-x\right)^{2}\right)^{1 / 2}\right\} .
\end{aligned}
$$

Further, by (3), Lemma $1, \mathrm{c})$ and $x \in\left(0, \frac{1}{n}\right]$, we obtain

$$
\begin{aligned}
& \sum_{k=0}^{n-1} p_{n-1, k}\left(r_{n}(x)\right)\left(\frac{k}{n}-x\right)^{2}=\left(\frac{n-1}{n}\right)^{2} \sum_{k=0}^{n-1} p_{n-1, k}\left(r_{n}(x)\right)\left(\frac{k}{n-1}\right)^{2} \\
& \quad-2 x \frac{n-1}{n} \sum_{k=0}^{n-1} p_{n-1, k}\left(r_{n}(x)\right) \frac{k}{n-1}+x^{2} \sum_{k=0}^{n-1} p_{n-1, k}\left(r_{n}(x)\right) \\
&=\frac{\left(\frac{n-1}{n}\right)^{2}\left[r_{n}^{2}(x)+\frac{1}{n-1} r_{n}(x)\left(1-r_{n}(x)\right)\right]-2 x \frac{n-1}{n} r_{n}(x)+x^{2}}{=} \frac{(n-1)(n-2)}{n^{2}} r_{n}^{2}(x)+\frac{n-1}{n}\left(\frac{1}{n}-2 x\right) r_{n}(x)+x^{2} \\
& \leq \frac{(n-1)(n-2)}{n^{2}} r_{n}^{2}(x)+\frac{n-1}{n}\left(\frac{1}{n}+2 x\right) r_{n}(x)+x^{2} \\
& \leq \frac{(n-1)(n-2)}{n^{2}} \frac{1}{n^{2}}+\frac{n-1}{n}\left(\frac{1}{n}+\frac{2}{n}\right) \frac{1}{n}+\frac{1}{n^{2}} \\
& \leq \frac{1}{n^{2}}+\frac{3}{n^{2}}+\frac{1}{n^{2}}=\frac{5}{n^{2}} .
\end{aligned}
$$

Using the inequality $(a+b)^{2} \leq 2\left(a^{2}+b^{2}\right)$ with $a$ and $b$ real numbers, (16) and (3), we obtain

$$
\begin{aligned}
& \sum_{k=0}^{n-1} p_{n-1, k}\left(r_{n}(x)\right)\left(\frac{k+1}{n}-x\right)^{2} \\
& \leq 2 \sum_{k=0}^{n-1} p_{n-1, k}\left(r_{n}(x)\right)\left(\frac{k}{n}-x\right)^{2}+2 \sum_{k=0}^{n-1} p_{n-1, k}\left(r_{n}(x)\right) \frac{1}{n^{2}} \\
& \leq \frac{10}{n^{2}}+\frac{2}{n^{2}}=\frac{12}{n^{2}} .
\end{aligned}
$$

Combining (15), (16) and (17), we get

$$
\left|\varphi(x)\left(V_{n} g\right)^{\prime}(x)\right| \leq 4 n\left\|\varphi g^{\prime}\right\|\left(\frac{\sqrt{12}}{n}+\frac{\sqrt{5}}{n}\right) \leq 23\left\|\varphi g^{\prime}\right\| .
$$


Let $x \in\left[\frac{1}{n}, 1\right)$. For $g \in W(\varphi)$, by (1), (4), (12), Lemma 1, a), (9), Hölder's inequality, (4) and Lemma 1, c), d), e), we find that

$$
\begin{aligned}
& \left|\varphi(x)\left(V_{n} g\right)^{\prime}(x)\right|=\varphi(x)\left|\left(V_{n} g\right)^{\prime}(x)-g(x)\left(V_{n} e_{0}\right)^{\prime}(x)\right| \\
& =\varphi(x)\left|\sum_{k=0}^{n} p_{n, k}^{\prime}\left(r_{n}(x)\right) r_{n}^{\prime}(x)\left[g\left(\frac{k}{n}\right)-g(x)\right]\right| \\
& =\varphi(x) r_{n}^{\prime}(x)\left|\sum_{k=0}^{n} p_{n, k}^{\prime}\left(r_{n}(x)\right) \int_{x}^{\frac{k}{n}} g^{\prime}(u) d u\right| \\
& \leq 2 \varphi(x)\left|\sum_{k=0}^{n} \varphi^{-2}\left(r_{n}(x)\right)\left(k-n r_{n}(x)\right) p_{n, k}\left(r_{n}(x)\right) \int_{x}^{\frac{k}{n}} g^{\prime}(u) d u\right| \\
& \leq 2 \varphi(x) \varphi^{-2}\left(r_{n}(x)\right) \sum_{k=0}^{n}\left|k-n r_{n}(x)\right| p_{n, k}\left(r_{n}(x)\right)\left|\int_{x}^{\frac{k}{n}}\right| g^{\prime}(u)|d u| \\
& \leq 4 \varphi^{-2}\left(r_{n}(x)\right)\left\|\varphi g^{\prime}\right\| \sum_{k=0}^{n}\left|k-n r_{n}(x)\right|\left|\frac{k}{n}-x\right| p_{n, k}\left(r_{n}(x)\right) \\
& \leq 4 n \varphi^{-2}\left(r_{n}(x)\right)\left\|\varphi g^{\prime}\right\|\left(\sum_{k=0}^{n}\left(\frac{k}{n}-r_{n}(x)\right)^{2} p_{n, k}\left(r_{n}(x)\right)\right)^{1 / 2} \\
& \times\left(\sum_{k=0}^{n}\left(\frac{k}{n}-x\right)^{2} p_{n, k}\left(r_{n}(x)\right)\right)^{1 / 2} \\
& =4 n \varphi^{-2}\left(r_{n}(x)\right)\left\|\varphi g^{\prime}\right\|\left(V_{n}\left(e_{2} ; x\right)-2 r_{n}(x) V_{n}\left(e_{1} ; x\right)+r_{n}^{2}(x) V_{n}\left(e_{0} ; x\right)\right)^{1 / 2} \\
& \times\left(V_{n}\left(e_{2} ; x\right)-2 x V_{n}\left(e_{1} ; x\right)+x^{2} V_{n}\left(e_{0} ; x\right)\right)^{1 / 2} \\
& =4 n \varphi^{-2}\left(r_{n}(x)\right)\left\|\varphi g^{\prime}\right\|\left(x^{2}-r_{n}^{2}(x)\right)^{1 / 2}\left(2 x^{2}-2 x r_{n}(x)\right)^{1 / 2} \\
& =4 \sqrt{2} n \varphi^{-2}\left(r_{n}(x)\right)\left\|\varphi g^{\prime}\right\|\left(x+r_{n}(x)\right)^{1 / 2} \sqrt{x}\left(x-r_{n}(x)\right) \\
& \leq 4 \sqrt{2} n \varphi^{-2}\left(r_{n}(x)\right)\left\|\varphi g^{\prime}\right\| \sqrt{2 x} \sqrt{x} \frac{2}{n}(1-x)=16 \frac{\varphi^{2}(x)}{\varphi^{2}\left(r_{n}(x)\right)}\left\|\varphi g^{\prime}\right\| \\
& =16 \frac{x}{r_{n}(x)} \frac{1-x}{1-r_{n}(x)}\left\|\varphi g^{\prime}\right\| \leq 32\left\|\varphi g^{\prime}\right\| \text {. }
\end{aligned}
$$

Finally, we have $\varphi(0)\left(V_{n} g\right)^{\prime}(0)=0=\varphi(1)\left(V_{n} g\right)^{\prime}(1)$. Hence, by (18) and (19), we obtain $\left\|\varphi\left(\mathrm{V}_{\mathrm{n}} \mathrm{g}\right)^{\prime}\right\| \leq 32\left\|\varphi \mathrm{g}^{\prime}\right\|$, which completes the proof.

The next result is a weak-type version of the Berens-Lorentz lemma (see [1, 
p. 312, Lemma 5.2]).

Lemma 3 Let $\phi:[0, a] \rightarrow[0, \infty)$ be an increasing function with $\phi(0)=0$ and $0<\alpha<1$. If $0<a \leq 1$, then the inequalities

$$
\phi(a) \leq C_{5} a^{\alpha}
$$

and

$$
\phi(x) \leq C_{5}\left(y^{\alpha}+\frac{x}{y} \phi(y)\right), \quad 0 \leq x \leq y \leq a
$$

imply for some $\mathrm{C}_{6}=\mathrm{C}_{6}(\alpha)>0$ that

$$
\phi(x) \leq \mathrm{C}_{6} \mathrm{C}_{5} \mathrm{x}^{\alpha}, \quad 0 \leq \mathrm{x} \leq \mathrm{a} .
$$

Proof. Following the proof of Lemma 5.2 in [1, p. 312], it is easy to prove our result taking into account the slight modification on $\alpha$. For completeness we give the proof.

For $0<\mathrm{q}<1$, we define $x_{k}=q^{k} a, k=0,1,2, \ldots$ If we take $C \geq 1$, then (20) implies (22) for $x=x_{0}$. We prove (22) for all $x=x_{k}$ by induction. Let $\phi\left(x_{k}\right) \leq \mathrm{CC}_{5} \mathrm{x}_{\mathrm{k}}^{\alpha}$, then, by $(21)$,

$$
\phi\left(x_{k+1}\right) \leq C_{5}\left(x_{k}^{\alpha}+q \phi\left(x_{k}\right)\right) \leq C_{5}\left(1+q C C_{5}\right) x_{k}^{\alpha} \leq C_{5} C x_{k+1}^{\alpha},
$$

provided $1+\mathrm{qCC}_{5} \leq \mathrm{Cq}^{\alpha}$. To achieve this, we first take $\mathrm{q}$ so small that $\mathrm{q}^{\alpha}>\mathrm{C}_{5} \mathrm{q}$, because $0<\alpha<1$, and then $\mathrm{C}$ sufficiently large. After this, for any $0<x<a$, we select a $k$ with $x_{k+1} \leq x \leq x_{k}$ and get with $C_{6}:=\mathrm{Cq}^{-\alpha}$ the estimations $\phi(x) \leq \phi\left(x_{k}\right) \leq \mathrm{CC}_{5} x_{k}^{\alpha} \leq \mathrm{C}_{6} \mathrm{C}_{5} x^{\alpha}$.

In the next theorem we establish the converse result. We set $C_{01}[0,1]=\{f \in$ $C[0,1]: f(0)=f(1)\}$.

Theorem 2 For $\mathrm{f} \in \mathrm{C}_{01}[0,1], 0<\alpha<1$ and $\mathrm{V}_{\mathrm{n}}$ defined by (1)-(2), the estimation

$$
\left\|V_{n} f-f\right\| \leq C_{7} n^{-\alpha / 2}, \quad n=1,2, \ldots
$$

implies $\omega_{\varphi}^{1}(f ; \delta) \leq \mathrm{C}_{8} \delta^{\alpha}, 0<\delta \leq 1$.

Proof. The proof is based on Lemma 3 with $\phi(t)=\omega_{\varphi}^{1}(f ; t), t \in[0,1]$. For $f \in \mathrm{C}_{01}[0,1]$, by Lemma $\left.1, \mathrm{~b}\right)$, we have

$$
\begin{aligned}
\left(V_{1} f\right)(x) & =p_{1,0}\left(r_{1}(x)\right) f(0)+p_{1,1}\left(r_{1}(x)\right) f(1)=\left(1-x^{2}\right) f(0)+x^{2} f(1) \\
& =f(0)+x^{2}(f(1)-f(0))=f(0) .
\end{aligned}
$$


Therefore, by (5), $\omega_{\varphi}^{1}\left(f-V_{1} f ; t\right)=\omega_{\varphi}^{1}(f ; t), t>0$. Hence, due to (5) and (23),

$$
\omega_{\varphi}^{1}(f ; 1)=\omega_{\varphi}^{1}\left(f-V_{1} f ; 1\right) \leq 2\left\|f-V_{1} f\right\| \leq 2 C_{7} .
$$

Let $x \in[0,1]$ and $h>0$ such that $x \pm \frac{h}{2} \in[0,1]$, and let $\Delta_{h}^{1} f(x)=f\left(x+\frac{h}{2}\right)-$ $f\left(x-\frac{h}{2}\right)$. Then, by $(23)$,

$$
\begin{aligned}
& \left|\Delta_{h}^{1} f(x)\right| \leq\left|\Delta_{h}^{1}\left(f-V_{n} f\right)(x)\right|+\left|\Delta_{h}^{1}\left(V_{n} f\right)(x)\right| \\
& \quad \leq 2\left\|f-V_{n} f\right\|+\left|\Delta_{h}^{1}\left(V_{n} f\right)(x)\right| \leq 2 C_{7} n^{-\alpha / 2}+\left|\Delta_{h}^{1}\left(V_{n} f\right)(x)\right| .
\end{aligned}
$$

Using (6), we can choose $g=g_{\delta} \in A . C_{. l o c}[0,1]$ such that $\|f-g\| \leq C_{9} \omega_{\varphi}^{1}(f ; \delta)$ and $\left\|\varphi g^{\prime}\right\| \leq C_{10} \delta^{-1} \omega_{\varphi}^{1}(f ; \delta)$. Hence, in view of Lemma 2 ,

$$
\begin{aligned}
& \left|\left(V_{n} f\right)^{\prime}(x)\right| \leq\left|\left(V_{n}(f-g)\right)^{\prime}(x)\right|+\left|\left(V_{n} g\right)^{\prime}(x)\right| \\
& \quad \leq 8 \sqrt{n} \varphi^{-1}(x)\|f-g\|+32 \varphi^{-1}(x)\left\|\varphi g^{\prime}\right\| \\
& \quad \leq 8 \sqrt{n} C_{9} \varphi^{-1}(x) \omega_{\varphi}^{1}(f ; \delta)+32 C_{10} \delta^{-1} \varphi^{-1}(x) \omega_{\varphi}^{1}(f ; \delta) \\
& \quad \leq C_{11} \varphi^{-1}(x)\left(\sqrt{n}+\frac{1}{\delta}\right) \omega_{\varphi}^{1}(f ; \delta),
\end{aligned}
$$

where $C_{11}=8 C_{9}+32 C_{10}$. This implies that

$$
\left|\Delta_{h}^{1}\left(V_{n} f\right)(x)\right|=\left|\int_{x-\frac{h}{2}}^{x+\frac{h}{2}}\left(V_{n} f\right)^{\prime}(u) d u\right| \leq C_{11}\left(\sqrt{n}+\frac{1}{\delta}\right) \omega_{\varphi}^{1}(f ; \delta)\left|\int_{x-\frac{h}{2}}^{x+\frac{h}{2}} \frac{d u}{\varphi(u)}\right| .
$$

Because of $x \pm \frac{h}{2} \in[0,1]$, we have $x \in(0,1)$. Using (9), we obtain

$$
\begin{aligned}
\left|\int_{x-\frac{h}{2}}^{x+\frac{h}{2}} \frac{d u}{\varphi(u)}\right| & \leq\left|\int_{x}^{x+\frac{h}{2}} \frac{d u}{\varphi(u)}\right|+\left|\int_{x}^{x-\frac{h}{2}} \frac{d u}{\varphi(u)}\right| \\
& \leq 2 \varphi^{-1}(x) \frac{h}{2}+2 \varphi^{-1}(x) \frac{h}{2}=2 \varphi^{-1}(x) h .
\end{aligned}
$$

Hence, by (25), we get

$$
\begin{aligned}
\left|\Delta_{h}^{1} f(x)\right| & \leq 2 C_{7} n^{-\alpha / 2}+C_{11}\left(\sqrt{n}+\frac{1}{\delta}\right) \omega_{\varphi}^{1}(f ; \delta) 2 \varphi^{-1}(x) h \\
& \leq C_{12}\left\{n^{-\alpha / 2}+\left(h \sqrt{n}+\frac{h}{\delta}\right) \varphi^{-1}(x) \omega_{\varphi}^{1}(f ; \delta)\right\} .
\end{aligned}
$$

Replacing $h$ by $h \varphi(x)$ gives

$$
\left|\Delta_{h \varphi(x)}^{1} f(x)\right| \leq C_{12}\left\{n^{-\alpha / 2}+\left(h \sqrt{n}+\frac{h}{\delta}\right) \omega_{\varphi}^{1}(f ; \delta)\right\} .
$$


Now we choose $n \geq 1$ such that $\frac{1}{\sqrt{n}} \leq \delta \leq \frac{2}{\sqrt{n}}$, where $0<\delta \leq 1$. Then we find that

$$
\left|\Delta_{h \varphi(x)}^{1} f(x)\right| \leq C_{13}\left\{\delta^{\alpha}+\frac{h}{\delta} \omega_{\varphi}^{1}(f ; \delta)\right\}
$$

for all $x$ with $x \pm \frac{h}{2} \varphi(x) \in[0,1]$. Taking supremum over all $h$ with $0<h \leq t$, we obtain

$$
\omega_{\varphi}^{1}(f ; t) \leq C_{13}\left\{\delta^{\alpha}+\frac{t}{\delta} \omega_{\varphi}^{1}(f ; \delta)\right\}, \quad 0<t \leq \delta .
$$

Now (24) and (26) yield the assertion of our theorem by Lemma 3.

\section{References}

[1] R. A. DeVore, G. G. Lorentz, Constructive Approximation, Springer, Berlin, 1993.

[2] Z. Ditzian, V. Totik, Moduli of Smoothness, Springer, New York, 1987.

[3] J. P. King, Positive linear operators which preserve $x^{2}$, Acta Math. Hungar., 99 (2003), 203-208.

Received: June 3, 2019 